\title{
LOS SENTIDOS DE LA BROMA ENTRE LOS RARÁMURI DEL ALTO RÍO CONCHOS (CHIHUAHUA, MÉXICO)
}

\section{The Meaning of the Joke among the Raramuri of Alto Rio Conchos}

\author{
(Chihuahua, Mexico)
}

\author{
ABEL RODRÍGUEZ*
}

Fecha de recepción: 26 agosto de 2020 - Fecha de aprobación: 28 de abril de 2021

\section{Resumen}

Apoyado en la teoría del humor, y desde un punto de vista interpretativo de la cultura, este artículo tiene por objetivo analizar la broma entre los rarámuri del Alto Río Conchos de Chihuahua, México. Los datos etnográficos muestran la relevancia de la broma expresada en los contextos lingüístico, social y simbólico, en los cuales se puede constatar el comportamiento bromista de los rarámuri. Esto lleva al autor a concluir que, además de que la broma funciona como conjunción o disyunción en las relaciones sociales, su sentido último es reproducir formas de arbitrariedad cultural posibilitando así la conformación del "mundo al revés", ya que invierte y corrige las relaciones en favor del agente bromista individual o colectivo.

Palabras clave: broma; rarámuri; función; significados; México.

\section{Abstract}

Supported by theory of humor and from an interpretative point of view of culture, this article aims to analyze the joke among Raramuri of Upper Río Conchos in Chihuahua, Mexico. Ethnography data shows the relevance of joke expressed in linguistic, social and symbolic contexts, in which the joking behavior of the Raramuri can be verified. This leads the author to conclude that, in addition the joke works as a conjunction or disjunction in social relationships, its ultimate meaning is to reproduce ways of cultural arbitrariness, thus making it possible to shape the "upside down world", since it reverses and corrects relations in favor of individual or collective prankster.

Keywords: joke; Raramuri; function; meanings; Mexico.

\footnotetext{
* Dr. en Estudios Mesoamericanos. Académico Facultad de Ciencias Sociales y Humanidades, Universidad Autónoma de San Luis Potosí. Artículo enmarcado en el proyecto "Procesos sociales, históricos y contemporáneos de los pueblos indígenas del centro y norte de México" y en la colaboración con el Cuerpo Académico de Estudios Decoloniales (CAUASLP-271) de la misma facultad. Correo-e: abel.rodriguez@uaslp.mx
} 


\section{Introducción}

Al arribar a una comunidad rarámuri, el etnógrafo puede tener la impresión de que los también conocidos como tarahumaras son personas serias, agrestes, desconfiadas y sumamente reservadas ${ }^{1}$. Sin embargo, al pasar el tiempo y convivir cotidianamente con ellos se puede observar lo contrario. Rostros sonrientes que resultan de la broma entre sí y hasta con el externo. Por medio de la broma, la sociabilidad y un ambiente alegre entre los rarámuri quedan manifiestos tanto en la vida privada como pública, en contextos como la familia, la danza, el trabajo, la ritualidad y, sobre todo, durante las tesgüinadas (reuniones colectivas en torno al fermentado de maíz o tesgüino). La broma entre ellos es un elemento que ha sido muy poco tomado en cuenta por la mayoría de los etnógrafos, incluso entre los clásicos, quienes crearon la imagen de estas personas como sumamente serias, parcas, melancólicas o del todo reservadas, que necesitaban del alcohol para socializar entre sí. Es lo que estableció, por ejemplo, Zingg al decir que "tan acentuada es su timidez, que no hay exageración en afirmar que la vida social normal no podría prosperar sin la ayuda del tesgüino" (Bennett \& Zingg 1978 [1935], p. 66). En este mismo sentido, Bennett afirmó que la tesgüinada era "el mayor mecanismo socializante en la vida del tarahumara" (Bennett \& Zingg 1978 [1935], p. 500). Y Lumholtz aseveró que "en el curso ordinario de su existencia, el tarahumar no civilizado es demasiado vergonzoso y modesto [...] resultando que se conserve y se propague la raza, gracias a los efectos del tesgüino" (Lumholtz 1981 [1902], p. 345).
La etnografía más reciente apenas hace hincapié en el comportamiento bromista de los rarámuri interesándose válidamente en otros temas. Destacan, por ejemplo, estudios sobre la danza, en los que se menciona de paso la broma en este contexto (Acuña, 2006; Bonfiglioli, 1995); un estudio sobre el concepto de "alma" en el que se señala, tangencialmente, la relación entre este y el estar contentos (Merrill, 1992). Pero es Velasco (2006 [1983]), quien mediante un tratamiento de más de cien páginas sobre las fiestas rarámuri, sugiere que esta es "la cultura de la alegría".

Existe solamente un estudio, hecho por John Kennedy (1970a), que trata en específico "las bromas jocosas". También para este autor, esta conducta "está exclusivamente asociada con la tesgüinada [... .] esto es, en la atmósfera permisiva engendrada por el alcohol" (pp. 38, 58). Kennedy reportó, además, que entre los rarámuri este tipo de bromas aparecen solo cuando hay relaciones de parentesco y que "prevalece entre generaciones alternas y cuñados" (Kennedy, 1970a, pp. $38,42)$. No obstante, reconoció que en la vida cotidiana la mención del término muchímari entre aquellos que son "cuñados", un término que hace referencia a lo jocoso, "casi siempre provocará risas, o al menos sonrisas" (p. 38).

En otro sentido, Kennedy señaló que, si bien estas bromas podrían verse como una "falta de respeto permitida" y como una forma de catarsis que evita el conflicto, como indicaba la visión funcionalista, "estas relaciones, el drama cómico y el humor en general, son instituciones que fomentan la cohesión y armonía con la misma eficacia que las costumbres del control social" (Kennedy, 1970a, p. 65). Como se ve, en términos teóricos, ensanchó la visión funcionalista sobre el tema, fundada por Radcliff Brown. 
El énfasis de Kennedy (1970a), como él lo dice:

[...] es que las relaciones de broma pueden ser entendidas como un tipo de juego o "juego" las cuales tienden a tomar esta forma debido a la naturaleza de las sociedades a pequeña escala o analfabetas. Mi énfasis está puesto en las recompensas positivas de la sociabilidad, más que en los efectos negativos del control social y la evitación de conflictos inminentes, ideas que han dominado por mucho tiempo el pensamiento sobre este tema (p. 37). (Traducción propia) ${ }^{2}$

Por lo tanto, para la comprensión de la broma jocosa, Kennedy puso el énfasis en la recompensa positiva de la sociabilidad y no en los efectos negativos del control social que esta conducta puede tener. Para él, la broma jocosa crea profundos lazos de amistad y fortalece las relaciones de parentesco como puede ocurrir en sociedades de baja escala. Es por ello que, para autores como este, se ha dicho que el humor fue visto como parte de las relaciones de parentesco cuyo análisis, y no el del humor per se, ayudaba a entender la sociedad en estudio (Apte, 1983; Oring, 2008).

En lo siguiente, trataré de responder a la pregunta sobre los significados de la broma entre los rarámuri del Alto Río Conchos. Para ello, hablaré de la broma que se expresa en la actividad cotidiana y en algunos momentos de la ritualidad y la fiesta. Mis objetivos son los siguientes: 1) describir la broma entre los rarámuri en una diversidad de contextos lingüístico, social y simbólico-; 2) mostrar que el parentesco no es una barrera infranqueable para la expresión de la broma entre ellos; 3) explicar que, en este caso, la broma funciona como conjunción o como disyunción en las relaciones sociales, pues las incrementa 0 reduce, y 4) demostrar que el sentido último de la broma es reproducir las formas de arbitra- riedad cultural -como son el ejercicio del poder y la jerarquización social- ya que posibilita la conformación del "mundo al revés", invirtiendo y corrigiendo las relaciones en favor del agente bromista individual o colectivo.

En primer lugar, expondré mi metodología. En segundo lugar, desarrollaré mi perspectiva teórica. En tercer lugar, presentaré los datos etnográficos registrados en los contextos lingüístico, social y simbólico en los que he observado el comportamiento bromista entre estas personas. En cuarto lugar, concluiré con un análisis interpretativo de este fenómeno cultural entre los rarámuri. Aclaro que extiendo la información etnográfica de cada contexto en el que aparece la broma con la finalidad de evitar la orientación psicologista que tienden a tener las teorías socioantropológicas sobre este fenómeno. Como señaló Apte (1983), estas teorías han sido especulativas "porque no pueden ser respaldadas por validación empírica y experimental" (p. 207).

\section{Metodología}

Los datos etnográficos que aparecerán aquí son resultado de diversas estancias de trabajo de campo, principalmente con miras a sustentar una tesis doctoral en Estudios Mesoamericanos en el marco de una historia regresiva, que siguió como eje el simbolismo ritual del presente (Rodríguez, 2013). Dichas estancias fueron hechas en Chinéachi (diciembre de 2007 a junio de 2008) y Narárachi (abril de 2008, marzo-abril de 2009 y abril de 2010), aprovechando el hecho de que previamente había tenido la oportunidad de aprender la lengua de este pueblo en diversas rancherías rarámuri de Tewerichi (2000-2003), además de 
la experiencia de estancias de investigación anteriores en Norogachi (marzo-abril de 2004), Tewerichi (marzo-abril de 2005) y Narárachi (diciembre de 2006 - enero de 2007). Así también, algunos datos son producto del trabajo de campo realizado en distintos momentos del ciclo ritual, en Narárachi y Tewerichi, entre 2011 y 2016, tiempo en que fungí como coordinador de la Escuela de Antropología e Historia del Norte de México, en Creel, Chihuahua. Del mismo modo, el trabajo de campo más reciente (2018-2019), también en Narárachi y Tewerichi, me ha permitido complementar y corroborar datos aquí presentados.

Los datos han sido registrados en varios diarios de campo, así como en algunas grabaciones de audio y video, y en un archivo fotográfico. Los considero, de manera estratigráfica, en tres niveles de importancia. En el primero están los que, en su mayoría, me fueron confiados en diálogos informales durante eventos rituales y de la vida cotidiana, pero en especial durante las tesgüinadas que siguen a todo evento ritual y festivo, en las que la ritualidad continúa en diversas formas, incluyendo la broma, espacios que siempre he valorado como los mejores momentos para entrar en mayor confianza con los rarámuri, además, en su propia lengua (variante norte) ${ }^{3}$. Asimismo, en ocasiones me involucré como danzante y esto me permitió observar y escuchar de primera mano acciones y discursos bromistas entre jóvenes y adultos. Lo mismo ocurrió al apersonarme en la tienda de la cabecera ejidal cada domingo para conversar con algunos de los muchos rarámuri que acuden a comprar su despensa semanal después de haber acudido al "rezo" y escuchado los discursos y avisos de sus autoridades.
Estos momentos, y otros de caminatas entre rancherías, con guías rarámuri, constituyen el campo en el que creo haber logrado captar lo que Galinier (2001) Ilama "exégesis interna", ese "conglomerado de comentarios desordenados, de cantos, de 'pequeñas historias' y sobre todo de chistes, juegos de palabras, que circulan entre los mismos indígenas" (p. 457) sin la solicitud del antropólogo. En el segundo nivel están los datos que obtuve en las conversaciones formales con algunos rarámuri, con quienes, por diversas circunstancias, tuve y conservo la amistad. En un tercer nivel considero aquellos datos, los menos quizá, obtenidos en conversaciones algo más formales, a modo de entrevista abierta, con algunos interlocutores, y aquella información que me fue proporcionada por algunos externos de las comunidades rarámuri, pero que conviven con ellos. Siempre que lo consideré pertinente, retribuí a mis colaboradores del modo en que entre ellos practican el korima o la ayuda mutua como sistema de reciprocidad.

\section{Aclaración conceptual}

Me parece pertinente comenzar haciendo una aclaración conceptual entre lo que es el "juego" y lo que es la "broma", esto debido, principalmente, a que mis datos etnográficos así lo exigen. Al observar el comportamiento bromista entre los rarámuri pareciera ser que la broma es por lo común un juego, pero no siempre el juego es una broma. ¿Qué es, entonces, juego y qué broma?, ¿cómo se distinguen? Siguiendo a Huizinga (2007), la broma es, de ordinario, verbal $y$, aun cuando pudiera haber espectadores, "esta no necesariamente requiere de un público para tener expresión" (p. 39); por el contrario, 
el juego necesita espectadores. Además, el juego, debido a las reglas que lo enmarcan, "se mantiene fuera de las exigencias de la vida corriente" (Huizinga, 2007, pp. 21, 257) lo que no ocurre con la broma. Asimismo, a diferencia del juego, la broma se puede presentar espontáneamente en la actividad cotidiana y queda enmarcada en un campo semántico más amplio, como es el humor, en el cual entran, además, diversas formas en las que la broma se materializa como diversión, risa y chiste.

Por lo demás, no trataré aquí de entender qué es el humor de un modo lato, cuestión muy compleja y con muchas aristas, pues, por ejemplo, así como el humor puede apuntalar sistemas ideológicos, conservadores o subversivos, también puede tanto atemperar los ánimos como exaltarlos (Llera, 2004).

En el amplio campo semántico que abarca el humor destaca la broma, fenómeno que aparece en el acontecer de la actividad cotidiana integrándose al espacio de la sociabilidad. La broma disyunta o conjunta, en términos de la lógica formal, porque incrementa o disminuye las relaciones, además de fortalecerlas o debilitarlas (Abril, 1980). A veces "construye procesos de solidaridad e insolidaridad; une y a la vez separa [...] reproduciendo las formas de arbitrariedad cultural" (Infante, 2008, p. 259), como son las tropelías en el ejercicio del poder y la jerarquización social. Sin embargo, la broma no es un fenómeno exclusivo de la cultura, la sociedad, los valores, el parentesco, lo estético o lo psicológico. Como el humor, la broma es también "la combinación de campos, a los que se añaden elementos simbólicos que configuran un proceso complejo, dispuesto a provocar espacios de tensión y desequilibrio" (Infante, 2008, p. 259).
El humor, visto así, a través de la broma, es fuente principal de la risa, de ese gesto social que parece circular de la misma forma en todos los seres humanos o al menos de manera muy similar. No obstante, en cada grupo humano en que se presenta, este gesto también tiene variedad debido, principalmente, a las diversas "causas e instituciones [...] que en cada cultura se desarrollan" (Jáuregui, 2008, p. 61). A su vez, como vehículos de expresión de la broma aparecen la diversión y el chiste. El chiste, visto como un fenómeno social pluridimensional, es inherentemente dialógico porque casi siempre despierta la risa $u$ otras reacciones en un "otro" (Torres, 1997-1998). A través del chiste, la broma ridiculiza valores y pretensiones: "su dinámica se ordena a partir de la degradación -no siempre provocada- de ciertos valores expuestos mediante lo ridículo" (Infante, 2008, p. 259). Esta degradación de los valores del "otro" ocurre, así, mediante la risa, a través del chiste y el comportamiento bromista cuando estos fenómenos se relacionan con el poder y la jerarquización social imperante (López, 2008). Sin embargo, aun cuando el poder queda relativizado a través de la broma, este vuelve a aparecer en el lado opuesto imponiendo las formas de arbitrariedad cultural. La broma aplica una corrección, con la que aparece el efecto del "mundo al revés", una inversión de papeles que se vuelve contra quien la provocó (Bergson, 2011 [1900]).

Conjuntamente con lo anterior, consideraré que el vehículo último de la broma es el lenguaje. Me inspiro aquí en una de las ideas fundamentales de Sapir (1956), quien planteó que el lenguaje es resultado de un proceso cultural y social. De allí que, para alcanzar una mejor comprensión de un grupo humano, habría que aproximarse a los puntos de inter- 
sección entre el pensamiento, la lengua y las acciones sociales. Por lo tanto, mi aproximación a los datos ha sido hecha observando el sistema lengua-cultura y bajo la premisa de que "el lenguaje común y ciertos usos especializados de las palabras comunes constituyen el principal vehículo de las representaciones comunes de la sociedad" (Bourdieu, Chamboredon \& Passeron, 2008, p. 32). De acuerdo con Torres (1997-1998), esta consideración del humor como "juego comunicativo" me permitirá introducir un análisis de carácter pragmático para complementar la comprensión semántica del fenómeno humorístico que aquí presento, enfatizando la forma que este toma en la broma.

En un marco antropológico pretendo llevar a cabo un análisis interpretativo, por lo que consideraré la cultura como un conjunto ilimitado de contextos perceptibles (Wagner, 2018 [1975]) donde cada contexto es un "grupo de elementos simbólicos que se presentan juntos, ya formen una secuencia o una entidad reconocible" (p. 124). Cada grupo de símbolos, a su vez, forma parte de la experiencia y al mismo tiempo es construido, o "inventado", por ella. Es, además, una trama de significaciones, "un contexto dentro del cual pueden describirse todos esos fenómenos de manera inteligible, es decir densa" (Geertz, 2005 [1973], p. 27). Por ello, estimaré aquí que todo cuanto el ser humano hace o expresa es entendido como una urdimbre de símbolos susceptibles de ser interpretados. Estos símbolos, que también hacen presente lo no presente, se observan en un contexto determinado compuesto por el ethos-condiciones morales y estéticas- de una sociedad que, desde el punto de vista ideológico, puede mezclarse con una visión de las cosas (Geertz, 2005 [1973]). Este conjunto, ethos y cosmovisión, aceptado en este escrito, no es homogéneo. Por lo tanto, cuando hable de "los rarámuri" me estaré refiriendo, en específico, a aquellos que consideré mis colaboradores, a quienes observé y escuché, y no a la generalidad de este pueblo.

\section{Contexto etnográfico. \\ Los rarámuri del Alto Río Conchos}

\section{Los rarámuri}

Los rarámuri habitan la Sierra Tarahumara de Chihuahua, en el noroeste de México. Según cifras del Instituto Nacional de Estadística y Geografía (INEGI, 2015), actualmente viven ahí unos 73.856 hablantes de la lengua rarámuri. Ellos comparten la sierra con blancos y mestizos, pero también siguen siendo, aún hoy, vecinos de grupos con los que están emparentados lingüísticamente -los yuto nahuas-, como los pimas, los warijíos y los tepehuanes del norte. Aquí me referiré, al mencionar a Alto Río Conchos (en adelante ARC), al conjunto de cuatro comunidades rarámuri: Tewerichi, Narárachi, San José Bakiachi y Chinéachi. Estas tienen un sustrato histórico común, una variante lingüística compartida, un asentamiento por causas históricas y sociales comunes, una organización social muy similar y ritualidad con pocas variaciones (Rodríguez, 2013).

Una comunidad rarámuri en el ARC se caracteriza por la dispersión de numerosas rancherías a lo largo del río, cerca de un afluente de este o en alguna meseta que ofrezca un espacio para el cultivo. Cada ranchería puede contar entre dos y seis viviendas alejadas entre sí, conectadas por veredas. Ahí es donde primeramente se desarrolla la vida y la actividad cotidiana de estas personas. Estas 
comunidades (un conjunto de rancherías) se insertan, a su vez, en el mapa geopolítico mexicano formando un ejido (porciones de territorio rural de uso colectivo). Con población rarámuri, principalmente, estos ejidos se constituyen con distintas extensiones y número de población, siendo tal vez Narárachi (con 612 ejidatarios inscritos en su padrón) uno de los de mayores proporciones, incluso a nivel Sierra Tarahumara, y Chinéachi (con 72 ejidatarios inscritos), uno de los menos extensos. Casi siempre coincide que el ejidatario es un padre de familia y que esta está conformada por un promedio de seis miembros.

Cada ejido cuenta con una cabecera, constituida, por lo general, por un conjunto de construcciones: la tienda Diconsa o Liconsa, un internado, una clínica, un komerachi (cocina común y cárcel), una iglesia, un centro comunitario o maternal y algunas viviendas. Estas cabeceras concentran a los rarámuri provenientes de sus rancherías, cada domingo o días festivos, por motivos comerciales, económicos, educativos, jurídicos y/o religiosos. La altura promedio de estos centros es de 2.000 msnm (INEGI, 2009). Esta microrregión pertenece al municipio de Carichí, el cual se ubica, asimismo, en la región norte de la sierra chihuahuense.

Si bien en esta región la temperatura media es de 18 a $20^{\circ} \mathrm{C}$, en el invierno el termómetro puede llegar a descender hasta $-20 \stackrel{\circ}{ } \mathrm{C}$ y ascender en verano entre 25 y $30^{\circ} \mathrm{C}$. Como en casi toda la Tarahumara, la precipitación pluvial oscila entre los 550 y los 1.200 mm. Las lluvias que van de junio a septiembre son escasas, pero suficientes, en ocasiones, para la agricultura de temporal que los rarámuri practican en pequeños terrenos que ofrecen una cosecha considerablemente pobre. La diversidad vegetal en esta región es notable; predominan el pino, el encino, el táscate, el álamo, el fresno, el roble, la manzanilla y el madroño. Crecen, además, una serie de plantas comestibles (diversos tipos de quelite y hongos) y medicinales (chuchupate, anís y otras) (Sariego, 2002). La fauna de la región todavía aporta a la dieta de muchos rarámuri: conejos, ratón de campo, lagartijas, liebres, víboras de cascabel, ardillas, pavo salvaje y algunos venados. También abundan, yo mismo he avistado, aves como las güilotas, las aguilillas, los halcones, las auras y el pájaro carpintero pequeño. La pesca aporta, básicamente, bagre y trucha.

Los rarámuri en esta zona son agricultores, pastores de ganado caprino y vacuno en baja escala, artesanos y jornaleros. Sus principales cultivos y alimentos son la calabaza, el frijol y el maíz, pero la dieta se complementa con la caza, la recolección y la pesca-casi todos comparten los afluentes del río Conchos-. Asimismo, los alimentos enlatados (atún, sardina y sopas), la manteca, las galletas empaquetadas, el café y el maíz industrializado que pueden comprar en las tiendas de la cabecera ejidal forman parte, cada vez más, de la alimentación familiar. Con el maíz preparan el tesgüino, cerveza local empleada en toda reunión de trabajo colectivo y en las prácticas religiosas, contextos más que propicios para la sociabilidad y, ni qué decir tiene, para la expresión de la broma.

Algunos hombres fabrican instrumentos musicales, herramientas de trabajo y otros utensilios de madera. Algunas mujeres confeccionan cestos, ollas y ropa, a base de palmilla, barro, tela e hilos. En épocas previas a la siembra y la cosecha, hay quienes se emplean en la recolección de manzana o de tomate, en ranchos menonitas y mestizos, en la construc- 
ción de viviendas y hasta como mecánicos, traductores o empleadas domésticas en las cabeceras municipales más pobladas de la sierra o en las principales ciudades del estado de Chihuahua y de otros estados vecinos como Sonora y Sinaloa. El primer registro que se tiene de ellos es del jesuita catalán Joan Font, quien en 1607 describió ampliamente a los que él llamó los "taraumaros" (González, 1982, p. 155; 1987, p. 147).

\section{La broma y la búsqueda de sentidos entre los rarámuri}

\section{Contexto lingüístico}

En la lengua rarámuri el verbo re'éma, 'jugar', o el sustantivo re'ékiame, 'juego', son empleados para referirse al deporte; por ejemplo, a la práctica masculina que Ilaman rarajípama, 'correr la bola', o al deporte femenino rowéraka, 'correr los aros'. En estos juegos hay espectadores y determinadas reglas que los participantes deben acatar. Además, se hacen apuestas y, por ello, son también espacios de redistribución de riqueza. Por ser estos eventos "especiales", en la mayoría de las ocasiones en que se llevan a cabo se mantienen fuera de las exigencias de la vida ordinaria y esto los asemeja incluso a rituales. Asimismo, podemos escuchar expresiones como ¡rarajipobo! o ¡rowérabo!, 'vamos a correr la bola' o '...los aros', en las cuales está implícito el verbo 'jugar'. En estos casos, dicho verbo no tiene el sentido de bromear.

No obstante, el verbo 'jugar', re'éma, tiene también el significado de "diversión", como en el caso del juego de mesa llamado el 15 -para el cual se emplean dados- o el del cuatro, que se juega en sitios abiertos. Otro caso son los juegos infantiles como el del coyote, cuando los niños dicen: ¡basachi i’kíka re’épo!, ‘jvamos a jugar al coyote que muerde!'. Se trata de un juego muy usual en Tewerichi y Narárachi, en el cual los niños se divierten subiendo entre las ramas de un árbol y juegan al modo de nuestro "la traes". Los juegos como estos, que se llevan a cabo como parte de la actividad cotidiana, entre vecinos cercanos y lejanos, adultos, jóvenes o niños, tienen, además, un sentido muy cercano al de "bromear" porque la incesante risa y los chistes o las burlas debido a una equivocación, a una caída, a la tentativa de atrapar al otro y errar en el intento, crean siempre un entorno bromista.

Pero, más concretamente, existe el verbo ramuema, 'bromear', cuyo radical tiene amplia productividad. De este modo, existen voces como ramueéri, 'broma' o 'picardía'; ramuérema, 'hacer bromas a alguien'; ramúerame, 'bromista', o ramuérama, 'chiste'. Y así dicen, por ejemplo, Barintini we ramuérame jú, 'Valentín es muy bromista'4.

Existe, además, el término akareantiri, cuya raíz proviene del verbo akarema o 'mentir', que hace referencia específicamente a los chistes -verbales o con ademanes corporalesque provocan risa. Los rarámuri traducen el término akareantiri como 'medio mentira', cuyo sentido es bromear. A este se refieren cuando, por ejemplo, hay una noticia falsa que se desmiente de inmediato provocando risas. Por ejemplo, cuando el vendedor de la tienda dice un precio demasiado alto sobre un producto por el que se le ha preguntado, pero de inmediato corrige y enseguida dice el precio correcto precedido de un "no te creas", para indicar que bromeaba. 
Existe también la expresión pe echi siame, 'amenaza', que hace referencia a una persona de quien no se sabe si al hablar muy solemnemente está bromeando o está molesto sino hasta que ocurre su segunda reacción que será romper en risas o bien mostrar una verdadera molestia. Esto ocurre cuando una broma, entre al menos dos personas, parece haber llegado a un límite en el cual a quien se aplicó dicha broma se muestra molesto o bien demuestra que continuará con el ambiente de chacoteo. Existe también el término rasíame, 'grosero' o 'malcriado', usual entre los adultos, en especial cuando les parece excesivo el contenido sexual de la conducta bromista en alguien. $Y$ no falta el achíame, 'risueño' o 'el que ríe mucho', en el sentido de señalar a alguien que en "todo momento" parece alegre y bromista ${ }^{5}$.

El empleo de una terminología amplia para hacer referencia a la broma indica, por lo pronto, que el buen humor, la empatía y la guasa, así como, sin duda, las actitudes opuestas, están presentes en la sociabilidad rarámuri. Este patrón parece común al mundo indígena americano, o al menos así lo sugieren casos como el de los mapuche, quienes cuentan con un verbo 'reír' sorprendentemente productivo incluido, además, en algunos nombres personales (Salinas, 1991). También la lengua guaraní, al modo de los rarámuri, designa el humor con una amplia terminología (Salinas, 1996). Pero, ¿qué significado tiene la relevancia del humor en la lengua rarámuri? Para intentar responder a esta pregunta es necesario observar tanto el contexto social como el simbólico.

\section{Contexto social.}

\section{Parentesco}

En términos de parentesco, Kennedy reportó que las bromas jocosas se dan entre cuñados. Entre el esposo de un ego y la(s) hermana(s) de ego -el esposo llama a cada una de ellas upira o 'mi esposa'-, y entre la esposa de un ego y el(los) hermano(s) de ego -nombrados por ella kunara o 'mi esposo'- (Kennedy, 1970a, p. 39; 1970b). También pueden darse entre generaciones alternas -abuelos y nietos- (Kennedy, 1970a, p. 41) y entre hombres que son cuñados -muchímari o chiéri-.

El primero es un caso que yo mismo he constatado pero, de acuerdo con un colaborador, únicamente ocurre si desde tiempo atrás la pareja y sus familiares han tenido una relación cercana. Es decir, esta relación se da en aquellos matrimonios en los que desde muy pequeños las parejas y, por tanto, los cuñados y las cuñadas fueron vecinos. Si no ha sido así, la broma jocosa no aflora fácilmente. "Solo si hay confianza, pero no siempre es así", me explicó Nevares, un rarámuri adulto, casado y ahora viudo. Debo aclarar que Kennedy trabajó en la comunidad de Inápuchi, un reducto de rarámuri conocidos como "gentiles" porque nunca han aceptado a los misioneros católicos, y que mis datos fueron recogidos en una región de rarámuri pagótuame, es decir, 'rarámuri bautizados'. Sin embargo, ideológicamente no existen profundas diferencias entre ellos. Como lo ha probado la etnografía, el cristianismo es superficial incluso entre los rarámuri considerados hoy como cristianos porque piden el bautismo católico. Véase, por ejemplo, lo que dice Merrill (1992) sobre el sentido del término pagótuame: 
Las personas que actúan apropiadamente -que son amables con los otros, que cumplen con sus responsabilidades, que están bien dispuestos a compartir su comida y su fuerza de trabajo y se abstienen de ser agresivos- son caracterizados no solamente como "gente buena" (wé ga'rá pagótuame hú) sino como "buenos pensadores" (wé ga'rá nátame hú). (p. 104)

Asimismo, Merrill añade que "el hecho de haber recibido el sacramento del bautismo se considera irrelevante al hecho de que sean "buenas personas'” (p. 104, nota al pie 4). Esta es, sin duda, una conclusión aplicable a nuestra región de estudio, pues fuera de la petición de este sacramento para los infantes, ellos no solicitan nada más de la Iglesia católica (Rodríguez, 2013).

Asimismo, en toda la Sierra Tarahumara, al día de hoy, debido probablemente a que cada vez hay una mayor cantidad de matrimonios exógamos entre rarámuri de comunidades lejanas entre sí, situación favorecida quizá por el incremento de vías de acceso -brechas, caminos y carreteras-, esta relación de broma permitida entre cuñados de sexo opuesto parece ir desapareciendo. Esto último me ha sido sugerido por casos como el de Javier, un joven rarámuri casado en 2013 en Tewerichi, quien me comentó lo siguiente:

Yo soy de un rancho de Norogachi y mi esposa de Tewerichi [...] Tenemos tres años de casados [...] Yo no puedo hacer bromas como las que dices a mis dos cuñadas [no puede llamarles "esposas"] porque si lo hiciera a lo mejor sus esposos se enojan y no quiero que se enojen conmigo [...] a veces bromeamos cuando están haciendo tortillas pero muy poco [...] por ejemplo, un día que una cuñada hacía tortillas le dije "ya te puedes casar", aunque ya está casada, pero es que así decimos en Norogachi a las mujeres que saben hacer tortillas y comida, y que ya se pueden casar. (J. Rekiachi, comunicación personal, 1 de diciembre de 2019)
La familia sigue siendo, sin duda, el contexto en el cual los niños rarámuri asimilan la broma y aprenden a socializar con otros, a "inventar" y "recrear" su cultura. El padre enseña a sus hijos a bromear cuando lo hacen entre ambos. Una vez observé cómo un padre y su hijo bromeaban al cruzar una parte del río Conchos que pasa por Tewerichi y cuya corriente tenía bajo nivel, pero había que cruzarlo pasando sobre piedras dispuestas a modo de puente entre una y otra orilla. "¡Uyy, a que te caes!”, decía el padre bromeando a asustar al hijo. "A que no, el que se va a caer eres tú", decía el joven de unos 13 o 14 años de edad, y ambos reían.

El caso no es raro, más bien es común observar el comportamiento bromista entre niños, jóvenes y adultos como parte de las exigencias de la vida ordinaria. La broma es parte de la actividad cotidiana y aparece de manera continua lejos de la embriaguez. Entre mujeres sucede lo mismo, como me lo hizo saber una religiosa que fungió como enfermera en la clínica de Tewerichi en dos períodos (1974-2004 y 2008-2018): las madres bromean a sus hijas en casa diciéndoles, por ejemplo, ma ke rimúa eyena rejói yúa, 'ya no sueñes tanto con los hombres' (O. Magallanes, comunicación personal, 20 de enero de 2018), broma que, por lo común, hace que ambas rían en los momentos en que las jóvenes se activan en las labores del hogar. De esta manera, ellas aprenden los significados que puede tener el hecho de "soñar con un hombre" -noviazgo, matrimonio, maternidad, hijos, trabajos del hogar, parentela, etc.-.

A partir del aprendizaje familiar, la broma se orienta, culturalmente, de modo similar a como ocurre entre los muina de Colombia, para quienes es incluso una "manifestación de la conciencia viva de la cultura" (Tobón, 2016, 
pp. 181, 198). No es extraño que los abuelos influyan en los nietos aprendices de su cultura y del comportamiento bromista. Es común, por ejemplo, que el abuelo bromeé con su nieto diciéndole repetidamente 'mi suegro', kene siá, y que el niño comience a comprender asuntos del parentesco, como el de 'ser suegro'. Y aunque yo nunca observé que la abuela y el nieto bromearan jocosamente durante la tesgüinada, como señala Kennedy que ocurre, de acuerdo con los colaboradores esta relación también es usual.

Lo que sí observé en varias ocasiones fue que algunas madres llamaran ke ne moné, es decir, 'mi yerno', a determinado joven rarámuri casadero, haciéndole entender de este modo y entre risas que contaba con su beneplácito para que pudiera cortejar a una de sus hijas o que determinada hija así lo deseaba. Esta broma la observé en algunas tesgüinadas y en otras ocasiones de la vida ordinaria, con lo que ha me quedado claro, al día de hoy, de que se trata de un dispositivo que da cauce, efectivamente, al matrimonio. De este modo, en este contexto la broma funciona sobre todo, aunque no únicamente, como una conjunción en las relaciones sociales, pues crea espacios de solidaridad, amplía el parentesco y profundiza las relaciones entre los agentes.

En Tewerichi conocí a dos ancianos rarámuri, un hombre y una mujer, sin relación de parentesco, cuya charla siempre iniciaba con bromas. Tampoco faltaban en medio de la plática e incluso al terminar la charla. Al despedirse, ambos lo hacían en tono de broma y siempre sonriendo. En una ocasión, afuera de la tienda después de haber hecho sus compras, bromeaban sobre su edad avanzada, la pérdida de la fuerza para cargar sus bolsas y caminar cerro arriba, la probabilidad de caer o de que el burro de carga ya no hiciera caso a sus llamados porque no los escuchaba, pues ya no tenían la fuerza suficiente para gritarle como antes lo hacían. Este relato es un botón de muestra sobre cómo la broma es frecuente entre los rarámuri. Aunque es poco común que se mezclen mujeres y hombres en el ambiente de guasa, este caso no es raro. Los adultos mayores rarámuri de distinto sexo, coetáneos o próximos a serlo y no necesariamente parientes ni vecinos cercanos, bromean sobre la decadencia física, desgracia humana que quizá sólo puede ser superada un poco mediante la risa.

En otra ocasión presencié la siguiente broma: mujé anayáwari jú, echi ko ne nimí ku'íroma, 'ya eres antigua, por eso te voy a ayudar', le dijo un hombre de alrededor de 45 años a una mujer de unos 65, a quien ayudaría a cargar un costal con alimentos y a colocarlo sobre la espalda de ella. En esta broma hay un doble sentido en el uso del término anayáwari. Por un lado, este hace referencia a una persona mayor de edad o anciana; por el otro, alude a los Anayáwari, es decir, a los 'Antiguos rarámuri', sumamente importantes dentro de la cosmovisión indígena -desde el punto de vista ritual, social y político-. La enseñanza de los Antiguos, en términos éticos, es lo que todos los rarámuri actuales deben practicar. Con ello, quien expresa esta broma es alguien que relativiza la autoridad de los Antiguos, pero, al mismo tiempo, por ser un danzante mayor, infinidad de veces coordinador de los matachines y de otros danzantes en los distintos ciclos rituales, se le permite este comportamiento "iconoclasta". Entre los rarámuri, por lo general, nadie emplea el término Anayáwari como sinónimo de 'viejo' o 'anciano', para ello emplean los términos chérame si se trata de un hombre o weráame si de una mujer. 
Esto sugiere que la broma, además de conjuntar o disyuntar, tiene también el sentido de relativizar el poder; en este caso de las creencias y narrativas religiosas del grupo. Pero, además, el poder no solo queda relativizado a través de la broma, sino que reaparece en el lado opuesto y reproduce así el efecto del "mundo al revés", al ganar el bromista mayor autoridad.

Los rarámuri bromean haciendo chistes sobre la actividad cotidiana. Mientras se bebe una soda en la tienda o se comen algunas galletas, o si se encuentran en el camino dos rarámuri, hombres o mujeres. O bien, "cuando las mujeres lavan ropa a orillas del río o preparan la comida para los hombres que vuelven de la milpa o el acarreo de leña, ellas bromean y ríen abiertamente" (O. Magallanes, comunicación personal, 20 de enero de 2018). O bien, mientras los niños juegan básquetbol en la cancha del internado o mientras las niñas corren los aros. En todo momento los rarámuri emplean la broma como vehículo para la convivencia y comunicación interpersonal, y ríen continuamente. Este comportamiento bromista no es exclusivo de los eventos -familiares o comunitarios- que los congregan, sino también del día a día. La broma muestra aquí un sentido de conjunción de las relaciones sociales, conjunción en términos de la lógica formal, pues se trata de un conector que incrementa y profundiza las relaciones en el grupo.

Sin embargo, aunque la disyunción social, entendida como la disminución y la superficialidad de las relaciones mediante el dispositivo de la broma no es lo común, es también una posibilidad siempre latente en la sociabilidad (Abril, 1980). En este sentido, la broma puede presentarse también como parte de una disyunción social, o al menos como una introducción a esta. Ese fue el caso en una fuerte discusión que una vez presencié entre dos hermanos hombre y mujer adultos-. En el contexto de una tesgüinada, ella le preguntó, en tono de broma: “¿Qué milagro que nos visitas?”, a lo que él respondió, en un tono algo irónico, "pues he tenido mucho trabajo", y la discusión se desató. Ella reclamaba a su hermano no haber estado presente cuando, dos semanas atrás, su padre había muerto. La broma funcionó aquí como disyunción, pues el humor no siempre cimienta o edifica contextos solidarios, sino que a veces "construye procesos de [...] insolidaridad; une y a la vez separa" (Infante, 2008, p. 259).

\section{Tesgüinada y danza}

Un caso más de broma jocosa, según Kennedy, es el que se da entre hombres que son "cuñados". Agrega, además, que esta solo se observa durante las tesgüinadas y no en otro momento (Kennedy, 1970a, p. 41) 6 . De acuerdo con mis colaboradores, el término 'cuñado', en rarámuri muchímari, no es estrictamente un término de parentesco, como Kennedy asegura, pues se emplea tanto en el ámbito de la relación indicada como en la relación de pura amistad y confianza que puede haber entre dos rarámuri solteros que no son cuñados estrictos. Al respecto, un colaborador me dijo:

La palabra 'cuñado' puede usarse en forma de broma siempre y cuando a quien le dices tenga hermanas. O se le puede decir de verdad si está casado con tu hermana o tú te casaste de verdad con su hermana [... o puede decirse 'cuñado' de a mentira [de broma] si le tienes confianza a un amigo o si te gusta su hermana y te quieres casar con ella [...] o si tú le gustas a alguien para 'cuñado', pues te pueden decir cuñado [...] se puede decir muchímari o chiéra, es lo mismo, pero no siempre es un pariente de verdad. (J. Rekiachi, comunicación personal, 1 de diciembre de 2019) 
Las tesgüinadas son reuniones motivadas por ritos o ceremonias o bien por el trabajo colectivo -siembra, cosecha, construcción de viviendas o graneros, etc.-, que resultan en muchas ocasiones ser parte de las exigencias de la vida cotidiana. En estas, la bebida se considera como pago por haber colaborado con el organizador del evento o simplemente por haber sido invitado o invitada a este. En este contexto es probable que la broma de connotado carácter sexual surja de forma más espontánea, así como que el tono de la risa torne en carcajada más fácilmente. Pero estas formas no están exentas de la vida cotidiana, lógicamente pueden ser expresadas con mayor facilidad debido a la desinhibición que causa el consumo del alcohol. Esto es lo que sin duda ha hecho pensar a muchos que los rarámuri son sociables solo en medio de las borracheras. Por eso Bennett decía que en la influencia de la leve borrachera desaparecían "las barreras del silencio y la reserva. Todo el mundo habla, ríe y fuma" (Bennett \& Zingg, 1978 [1935]), p. 500).

Debido a la amplia concurrencia que suele haber, se arreglan asuntos e incluso puede ser la ocasión de enlaces matrimoniales que lleva a cabo el mayora, cargo comunitario autorizado para esto. Durante estas reuniones, el grupo de hombres y el de mujeres no se mezclan fácilmente y cada uno bebe por separado. Aunque esto no evita que, en algún momento, en especial algún hombre, se acerque al grupo de féminas y las interpele, bromeando, en unirse a ellas a platicar.

Dado que el fermentado del maíz es diurético, una broma común entre los hombres se expresa en el momento en que alguno dice: "ya vuelvo, voy a orinar", lo que motivará una serie de chacotas al susodicho sobre su "imposibi- lidad" para orinar de pie, "como los hombres". Esto lo dirán algunos de sus confidentes o acompañantes del momento, entre quienes puede haber parientes y no parientes. En este contexto, una broma frecuente entre mujeres es la que escuché que se hace a quien reparte la bebida y a quien todas las presentes le dicen 'hija' o 'hijita', en tono de broma, tengan o no parentesco con aquella que distribuye el licor. El sentido de esta broma es llamar a la joven que reparte la bebida para que se acerque a ofrecer el etílico.

Otra de las situaciones en que aparece la broma entre los rarámuri es la danza. Si bien esta se realiza siempre en el contexto de la ritualidad, contexto que está fuera de las exigencias de la vida ordinaria, la danza se presta para que el comportamiento bromista asome como en pocos ámbitos. Durante la Semana Santa, el comportamiento bromista es visible entre soldados y fariseos, los dos grupos antagónicos de danzantes ${ }^{7}$. Ahí, la broma de uno a otro grupo, así como entre los integrantes de cada bando y en especial de los fariseos hacia las mujeres que los observan, es muy común. Estos las conminan a ponerse en movimiento hacia la iglesia en determinados momentos, empleando para ello sus espadas hechas de madera dando paso así a una especie de broma ritual.

El grupo de los fariseos confecciona un "Judas" que después llevan en hombros mientras danzan. El muñeco tiene la particularidad de vestir a la usanza de los hombres blancos, además de que le sobresale un enorme pene hecho de madera que motiva risas al por mayor. En algún momento de la danza, la formación en filas se presta para que alguno bromee jocosamente con su colega de adelante simulando el coito. Puedo asegurar que esta broma no 
necesariamente se hace entre dos hombres que son cuñados, como afirmó Kennedy. La he observado al menos en una decena de ocasiones y no necesariamente entre cuñados, aunque sí entre pares de confianza. Tampoco había alcohol de por medio, pues se evita la ebriedad mientras dure la danza.

Quienes llevaron a cabo esta broma eran jóvenes no mayores de 20 años y lo hacían, parece, porque la situación casualmente se prestaba para ello. En otros casos que observé, en las filas de danzantes matachines, quienes participan en otro ciclo festivo, entre finales de abril y principios de enero, probablemente había la esperanza de ser cuñados en un futuro. $O$ bien, al menos, se trataría de una broma jocosa que ciertamente fortalecería los lazos de amistad, como Kennedy propuso, pues así me lo sugerían tanto la aceptación de la broma como las risas que esta despertaba en los partícipes y de otros a su alrededor. Sin despreciar la explicación catártica que pueda tener como desfogue, la broma funcionaba de nuevo como una conjunción de relaciones al profundizar la sociabilidad entre los agentes del entorno.

\section{Aplicación de justicia}

Aunque la impartición de justicia es parte de la actividad ordinaria de las autoridades rarámuri, los juicios no son frecuentes y se realizan en muy contadas ocasiones. Por lo general, estas personas son muy pacíficas y no trasgreden la etiqueta social fácilmente. Estos eventos pueden llevarse a cabo en especial los domingos, después del nawésari de las autoridades. Estos 'consejos' o 'discursos' recuerdan a niños y adultos, entre otras cosas, las enseñanzas de los Antiguos, las reglas de convivencia social o avisan sobre asuntos comunitarios. Al término de estos discursos, la máxima autoridad indígena de la comunidad puede ordenar que soldados y capitanes acerquen a acusados y acusadores. El resto de los asistentes representa a la comunidad ante la cual se lleva a cabo la impartición de justicia; las mujeres y niños menores se ubican del lado derecho de las autoridades y los hombres del lado izquierdo.

En una ocasión presencié un juicio en el cual el director de la escuela-internado de Tewerichi acusaba a un grupo de niños de haber ingresado a la bodega en que resguardaban la despensa. Luego de que los tres gobernadores indígenas hubieran ofrecido sus discursos para los rarámuri asistentes, el profesor Santiago, acompañado de las dos cocineras, además de la encargada del aseo del edificio y de un grupo de nueve niños de quinto y sexto de primaria, abordó al gobernador mayor y a su séquito de colaboradores -dos gobernadores menores, dos capitanes y dos soldados-. El profesor expuso la siguiente queja: La noche de jueves a viernes de la semana anterior, el grupo de niños que lo acompañaba había ingresado a la despensa y se había comido varios paquetes de galletas. Además, habían tomado latas de atún y, no conformes con comerlas cerca del internado, habían tirado el latón en los tambos usados para la basura de la misma escuela. La acusación incluía que los niños no habían respetado la privacidad del lugar, aun cuando desde el inicio de su estancia conocían el reglamento escolar, así como que hubieran dejado abierta la puerta de la bodega, lo que permitió que por la mañana algunos canes entraran a completar el botín comiendo paquetes de maíz en polvo para hacer tortillas. 
Terminada la exposición de quejas, el gobernador mayor llamó a los capitanes para que escoltaran al grupo de niños y los ubicaran frente a esta autoridad, la que pidió al profesor y sus acompañantes adultas que se pusieran al centro del patio para tener un careo con los impúberes. Entretanto, mujeres y hombres, de forma paulatina, fueron guardando silencio. Fue entonces que la autoridad mayor tomó la palabra y comenzó a arengar a los niños, quienes parecían un poco asustados. Al término de su discurso, el gobernador mayor consultó a sus colegas sobre cuál sería un buen castigo para estos transgresores del orden. Se sentaron y comenzaron el diálogo, ora entre gobernadores, ora entre el gobernador mayor y los quejosos.

Esto se llevó a cabo con dos silencios intercalados, mutismos que hicieron aparecer las bromas entre los hombres asistentes. En uno de estos silencios, un hombre adulto bromeó diciendo que uno de los niños era muy gordo debido, seguramente, al consumo de las galletas robadas (muchas risas). Otro joven bromeó diciendo que uno de los niños era hijo de un fulano, no asistente en ese momento a la reunión, que tenía fama de comelón de galletas (pocas risas). El asunto es que después de cada silencio-broma aparecía la risa, incluso entre las autoridades, y la broma funcionaba como una especie de conector lógico en el diálogo y como una conjunción de agentes que, cada uno en su rol, continuaron el juicio hasta el final.

Otro caso fue el de un joven, a quien llamo aquí Oscar, acusado de hostigar a una mujer viuda durante una tesgüinada. En este caso, el procedimiento fue similar al anterior, el gobernador mayor llamó al capitán, este a dos soldados y estos, al momento, ubicaron a Oscar y a la mujer frente a la autoridad mayor.
El gobernador tomó la palabra y comenzó a cuestionar al joven sobre su conducta preguntándole si acaso lo había hecho en estado de embriaguez. Esta cuestión es muy importante para los rarámuri porque es un elemento atenuante que disminuye la responsabilidad del transgresor. En este caso los silencios fueron más frecuentes y uno de estos llegó al límite cuando se le pidió a la mujer adulta que explicara el porqué de su acusación.

Cuando la mujer terminó de hablar, y el silencio se extendía, una primera broma se escuchó en el grupo de hombres. Un viejo de pelo blanco dijo: "yo creo que Oscar aprendió este comportamiento de su padre, quien tenía fama de perseguir mujeres", lo que despertó risas entre todos los hombres presentes, incluyendo a las autoridades y a algunas mujeres que alcanzaban a escuchar. A continuación, fluyó el diálogo entre el gobernador mayor y el acusado. Durante otro breve silencio, un hombre adulto, distinto al primero, dijo a un joven sentado junto a él: "sigue el ejemplo de Oscar y un día vas a estar ahí como él". En el entorno se escucharon risas solo entre algunos hombres. Otro adulto siguió la broma y le dijo al mismo chico: "o molesta a Oscar a ver si te hace caso" -risas solo de unos cuantos-, a lo que enseguida otro adulto respondió diciendo: "pero cómo no se casó en esa tesgüinada" -muchas risas-. Este juicio terminó en una aparente armonía al quedar conformes la acusadora y el acusado con los términos que propusieron las autoridades. El castigo para Oscar fue abastecer de leña a la viuda por los siguientes cuatro meses, con la consecuente multa de 100 pesos mexicanos (4.50 dólares estadounidenses actuales) cada vez que esta faltara y que la mujer lo volviera a denunciar. Esta cantidad parece mínima, pero en un contexto en el cual la fuente de trabajo la deben crear las propias manos, no es poca cosa. 
En los dos casos descritos la broma presenta distintas aristas. En primer lugar, siempre fue expresada por adultos o al menos jóvenes cercanos a la adultez. Llama la atención que, con ciertas bromas, todos o la mayoría ríen; pero, con otras, dichas por otros, solo algunos o muy pocos ríen e incluso algunos no ríen; también hay quienes no hacen bromas en estos casos. ¿Quién puede hacer bromas y qué es lo que otorga la autoridad para bromear? Las situaciones presentadas sugieren que la broma no puede ser expresada por cualquiera pues parece cierto que "nadie puede hacer una broma si no controla el contexto en que la hace" (López, 2008, p. 89). Sin duda, quienes mejor conocen el contexto casi siempre son los adultos y las bromas hechas por los mayores parecen tener especial repercusión y aceptación. Asimismo, quienes entre los adultos son bromistas parecen tener la prerrogativa de validar o invalidar las bromas de los jóvenes, no riendo o riendo moderadamente, como ocurre con los viejos chamanes secoya de la Amazonía ecuatoriana, cuya risa en respuesta a las bromas no es abierta y contagiosa, sino que impone autoridad sobre los neófitos (Cipolletti, 2000-2001).

Por lo tanto, conocer el contexto, tener ingenio para bromear pero, además, tener el reconocimiento de los demás, parecen ser las características de quien tiene la autoridad de emitir bromas, especialmente en contextos como los recién descritos. Todos los rarámuri conocen el contexto social de su comunidad en distinto nivel, pero no todos tienen el ingenio para hacer bromas y no todos cuentan con el reconocimiento social para hacerlo. El hecho de que no todos ríen cuando algunos expresan bromas parece demostrarlo, pero, más aún, nadie querría hacer el ridículo haciendo una broma con la cual nadie reiría; por eso, cada vez que en el grupo alguien hace una broma realiza una operación de riesgo, "que será rentable (incrementará su capital cultural) en caso de que la gente se ría, y será un fracaso (disminuirá su capital cultural) si no lo hace" (López, 2008, p. 80).

En segundo lugar, en situaciones como estas, durante los juicios, es difícil imaginar qué podría destrabar el silencio. Pareciera que, sin la broma, el fluir del juicio sería poco menos que imposible, la broma distensiona hasta en la guerra (Tobón, 2016). La broma, en estos casos, funcionaba nuevamente como una conjunción con la que se enlazaba un antes y un después del diálogo, pero también como una especie de "lubricante" y corrector de la situación, facilitador de la acción social como, siguiendo a Bergson, señala Abril (1980, p. 76).

\section{Contexto simbólico}

Hemos visto que la broma es recurrente entre los rarámuri, pero no representa un símbolo aislado. De ella se desprenden relaciones representativas, es decir, relaciones relevantes en cuanto conforman una trama mayor de elementos significativos (Geertz, 2005 [1973]). En otro espacio he probado la relevancia que tienen, en el pensamiento rarámuri, los conceptos de enfermedad y salud, y cómo estos han sido históricamente la base de la aceptación del bautismo y el crucifijo católicos, elementos que ellos han integrado a sus prácticas rituales (Rodríguez, 2010, 2013). Ahora bien, ¿cómo se relacionan estos conceptos, pilares del pensamiento rarámuri, con la broma?

Por un lado, entre los rarámuri estar contentos y tener soltura con la alegría, así como con la 
risa espontánea, podría ser una actitud identitaria. Esta actitud parece común al mundo indoamericano, o al menos así lo sugieren algunos casos como los ya citados más arriba sobre los guaraníes, los mapuche, los muina y los secoya. Por otro lado, mantener una disposición alegre es vital en una región agreste como es la Sierra Tarahumara. En ella la sequía, el frío, eventualmente las nevadas, y el arduo trabajo cotidiano, así como la continua invasión que desde diversas instituciones -religiosas, políticas y extractivistas- han padecido históricamente los rarámuri, convierten su contexto natural y social en un elemento a vencer con el buen humor y la salud, en contraposición con la tristeza y la enfermedad. Como ocurre entre los chácobo de Bolivia, también en esta visión de las cosas, "la tristeza puede comprometer a la persona de manera tal que hasta puede ocasionarle la muerte" (Balzano, 2001, p. 80).

Llama la atención que frente a la riqueza de la lengua rarámuri para referirse al buen humor, no exista el vocablo "serio" y que se llame kiri niraa, '(estar) tranquilo', a quien para nosotros muestra seriedad. Tal vez por ello, más que contraponer la seriedad a la alegría, ellos la contraponen, ante todo, a la tristeza. Para hablar de esta emoción los rarámuri emplean cuatro términos: sewé o ni'wíma para referirse a una tristeza aparente en alguien y o'móna o natemá para referirse a una aflicción profunda, como la pérdida de un ser querido o de la cosecha. Asimismo, el término 'salud' (estar bien de) se dice kánire (Lionnet, 1972, p. 103), la misma voz empleada para expresar que se está 'contento' o 'alegre'. Para el rarámuri tener salud es sinónimo de estar contento, riendo, bromeando. Para ellos, pensar demasiado un asunto puede provocar tristeza y esto podría acarrear enfermedad. "Si piensas mucho te vas a enfermar, ¡ánimo!", me dijo con una gran sonrisa, en una ocasión, un colaborador al verme en silencio y pensativo; para él, yo proyectaba tristeza.

Alejar la tristeza es muy importante porque en esto consiste alejar la enfermedad. ¿Cómo se aleja la tristeza? Con alegría, bromas y risas compartidas. Por lo tanto, en este modo de pensar, la tríada alegría, salud y vida se opone a la tríada tristeza, enfermedad y muerte. Esto nos ofrece una explicación sobre el sentido simbólico de la broma tan recurrente entre los rarámuri y niega que, a pesar de su gusto por el tesgüino, sean personas serias, parcas, melancólicas o extremadamente reservadas, que socializan únicamente si hay alcohol de por medio. La broma en las tesgüinadas llega, incluso, a tener un sentido ritual, como en aquellos momentos en los que pequeños grupos de hombres platican y bromean en torno a determinadas acciones del especialista ritual, a quien, después de bromear, respetan aún más. Para ellos, la broma y la risa tienen un sentido vital, pues ambas conforman los pilares de la salud, es decir, de la alegría que se desea hoy y siempre. Por ello, es pertinente decir que "el proceso humorístico se trata de un poder simbólico que bien puede contribuir en la construcción [...] en todo orden de cosas [incluso en la salud], no sólo estético o cultural, a largo plazo" (Infante, 2008, p. 263).

Asimismo, la broma entre los rarámuri saca a relucir algo más que "no está presente", mediante la burla y la broma pesada en la propia lengua indígena, contra aquellos o aquellas que los rarámuri sienten que actúan con talante de superioridad, arrogancia o apariencia de "muy educados". Antropólogos, monjas, curas, agentes de gobierno, profesores, directivos de educación y ONG han experimentado la broma en tono de aprecio o desprecio. En no pocos 
casos, los rarámuri tienden a hacer bromas a estos agentes, tan densas o pesadas como las llamadas bromas étnicas que los londinenses hacen a los irlandeses (Kravitz, 1977), a través de las cuales destaca en ellos una actitud de superioridad debido a que se encuentran empoderados por "conocer su contexto". Como el caso de esta broma hecha a un antropólogo, un desconocido recién llegado: "¿Tienes una hermana para que seamos cuñados?", hecha en lengua indígena. Entre las burlas, el aludido no sabía qué responder.

Otras preguntas a modo de broma pueden incluir temas relacionados con el físico o sobre lo repetitivo de un comportamiento, como cuando un joven, mientras yo platicaba con su padre, me dijo: "Escriba, ¿no va a escribir?", para luego reír profusamente. Con esto chacoteaba a propósito de la acción reiterativa que yo realizaba de escribir en mi libreta de campo. De este comportamiento bromista con los agentes externos, muchos mestizos serranos, a quienes en broma los rarámuri de la región llaman "hermanos mayores", pueden dar razón ${ }^{8}$. La broma, en estos casos, parece tener un sentido de resistencia cultural, incluso de reclamo, ya que parece hacer presente la invasión de sus tierras, la explotación de sus minas, la tala de su bosque, la imposición de megaproyectos turísticos o de infraestructura ${ }^{9}$, casos que han experimentado los rarámuri a lo largo de la historia y ante los cuales el último reducto de respuesta contra el poder y la jerarquización social impuesta parece ser la broma, dispositivo con el cual ellos "invierten" los papeles. Como dice Lagrou (2006) al estudiar el humor kaxiniwa "el poder provoca risa, así como la risa tiene poder" (p. 56).
Pero no es solo un placer estético lo que despierta la broma entre los rarámuri que se resisten a la imposición. En este caso, la broma disyunta creando un distanciamiento que vuelve a conjuntar las relaciones "invirtiendo" los papeles en cuanto se ha hecho una "corrección". De este modo, la broma induce al ridículo y deja a quien intenta imponerse, y por lo tanto provocándola, en una posición de supeditación, incluso cercana a la subordinación. A pesar de que esta corrección haya invertido los papeles tan solo por un momento, incita, puesto que es posible hacerlo, a su reiteración. La broma mantiene así a los rarámuri resistiendo.

\section{Conclusiones}

Además de la necesidad que tenemos de continuar recabando datos sobre la broma, como es el caso del albur, y de profundizar en la broma ritual entre los rarámuri, para ahondar en nuestras interpretaciones, de acuerdo con los contextos observados por ahora puedo enumerar las siguientes conclusiones:

1. Con los datos aquí presentados he probado que la broma es habitual en diferentes contextos -lingüístico, social y simbólico-, tanto en lo público como en lo privado, y he mostrado, además, que la conducta bromista es parte de las exigencias de la actividad ordinaria, aunque fuera de esta también es posible observar dicha conducta.

2. El análisis del contexto lingüístico hace evidente que entre los rarámuri la broma se expresa a través del lenguaje común. Pone de relieve que algunos usos especializados de las palabras constituyen el principal vehículo de las representaciones de esta sociedad y 
sugiere también la productividad de algunos elementos que conforman la terminología para referirse a las acciones en torno a este fenómeno social.

3. El contexto social demuestra que la broma está orientada culturalmente pues desde la infancia el rarámuri aprende, dentro del núcleo familiar, a socializar con la comunidad a través de la broma. Así, se conforma un ethos que se mezcla con una visión de las cosas, creando una identidad que cada individuo replantea según su inmersión, sus habilidades y el conocimiento que tenga del contexto social en el que se desenvuelve. De ahí que no todos los individuos bromeen y que algunos tengan mayor autoridad que otros para hacerlo.

4. Los casos presentados en torno al contexto social evidencian que el parentesco no impone reglas estrictas que impidan a los rarámuri expresar la broma. $Y$ aunque es verdad que no todo el mundo puede bromear con cualquiera, la broma aflora en las generaciones tanto alternas como continuas. Asimismo, la broma es un recurso comunicativo que puede posibilitar el acceso al parentesco con aquellos no parientes, como en el caso de las madres que buscan jóvenes casaderos para sus hijas, o como en el caso de hombres y mujeres que bromean durante las tesgüinadas o el de los jóvenes danzantes que se "amenazan" entre sí con bromas jocosas cuando solo hay amistad de por medio y el parentesco es únicamente una probabilidad futura.

5. La broma es un lubricante de las relaciones sociales tanto en los contextos comunitarios complejos y de tensión interpersonal como en la impartición de justicia o las relaciones interpersonales conflictivas. Se trata de un dispositivo que, en un primer nivel significativo, funciona como conjunción o disyunción porque puede incrementar o disminuir el orden de la sociabilidad que, a su vez, puede quedar fortalecido o debilitado.

6. Cuando he preguntado a mis colaboradores por qué bromean los rarámuri, la respuesta más común que me han dado es: mapu ke sewéka, 'para no estar tristes', o bien, mapu we kánireka eyena, 'para andar muy contentos -sanos-'. Más allá de la explicación que pueda tener la broma en términos de control social, como placer estético, por sus efectos catárticos o bien como refuerzo de los lazos de amistad entre ciertos agentes determinados por el parentesco, como señaló Kennedy, habría que añadir que los raramuri bromean porque este dispositivo permite reírse de la muerte y la vida, de la enfermedad y la salud, de los "otros" y de sí mismos, de los humanos y los dioses que se pretendan superiores, por su autoridad y poder -incluyendo a los Antiguos-. A través de la broma, el chiste y la risa, el individuo y la sociedad quedan aún más empoderados en su contexto al invertir continuamente el mundo "para estar contentos".

7. En un nivel más profundo de significación, la broma representa la probabilidad del "mundo al revés". Es decir, la broma puede devolver el control sobre las relaciones, pues a través de la superioridad en que queda el bromista se invierte la verticalidad en estas: el que está abajo puede estar arriba y viceversa. Es una muestra de cómo el poder fluye de arriba hacia abajo a través de este medio. Esto ocurre porque "las relaciones de poder han 
convertido el humor en un modo de mediación destinado a reproducir las formas de arbitrariedad cultural" (Infante, 2008, p. 259). Es decir, el ejercicio del poder, que puede formar parte de la arbitrariedad cultural, se hace aquí patente en cuanto el bromista, a través de la broma, tiene el control de la situación presente e impone su verdad. Por ello, en última

\section{Notas}

${ }^{1}$ Rarámuri (plural o singular) es un etnónimo mientras que tarahumara o tarahumaras es un exónimo.

2 "[...] is that joking relationships can best be understood as a type of game or "play" which tends to take the form it does because of the nature of small-scale non-literate societies. My emphasis is upon the positive rewards of sociability, rather than upon the negative effects of social control and avoidance of imminent conflict, ideas which have long dominated thinking on the subject".

3 "Las variantes de la lengua, en las diferentes áreas de la sierra, se ubican de la siguiente manera: 1) oeste, representada por las hablas localizadas al oeste de la barranca de Urique; 2) norte, que incluye a las hablas de Sisoguichi, Narárachi, Carichí, Ocórare, Pasigochi y Norogachi; 3) centro, representada por las hablas de la región de Guachochi; 4) cumbre, representada por las hablas localizadas entre las barrancas de Urique y Batopilas; y 5) sur, que incluye las hablas empleadas al sur de la barranca de la Sinforosa, al este de la región tepehuana, además de algunas subáreas consideradas como de transición" (Valiñas, 2002, pp. 260-261).

${ }^{4}$ En la lengua rarámuri no existe el fonema labiodental / $/$, de ahí Barintini.

${ }^{5}$ Si bien la terminología aquí presentada la recogí durante mi trabajo de campo, expresiones similares, en las distintas variantes de esta lengua, pueden corroborarse en el diccionario de Brambila (1976).

\section{Referencias bibliográficas}

Abril, G. (1980). Como gastar bromas. Revista Española de Investigaciones Sociológicas, 11, 75-89.

Acuña, A. (2006). Etnología de la danza en la Sierra Tarahumara. Granada: Universidad de Granada.

Apte, M. (1983). Humor research, methodology, and theory in anthropology. En Mcghee, P. E. \& Goldstein, J. H. (Eds.), Handbook of humor research, Vol. 1 (pp. 183-212). Nueva York, Berlín, Heidelberg, Tokio: Springer, Verlag.

Balzano, S. (2001). El chiste y su relación con las formas de instancia, la broma, como forma de mediación cultural entre los rarámuri, puede ser interpretada como este "mundo al revés", el mundo de la "inversión de papeles" (Bergson, 2011 [1900], p. 38), que se vuelve contra quien lo provoca porque ostenta o intenta ostentar superioridad, convirtiendo a la broma en una forma también de resistencia cultural.
${ }^{6}$ Descripciones e interpretaciones sobre la tesgüinada pueden verse en Kennedy (1963, 1970b) y Rodríguez (2009).

${ }^{7}$ Los soldados representan a los amigos de Jesús y, de manera más precisa, al bien. En contraposición, los fariseos representan a los amigos de Judas, al mal. Posteriormente, en el día a día, el grupo de soldados funge como parte de la estructura de autoridades que aplican justicia ante las faltas a la ética social en que algún rarámuri pueda incurrir.

${ }^{8}$ De acuerdo con Filiberto Gómez, "por ser hijos del Diablo, los chabóchi se sienten mayores de edad y ven a los indios 'como a cualquier chiquito', motivo que aprovechan los tarahumaras para preguntarse uno al otro en son de broma: '¿Qué andabas haciendo con tu hermano mayor?'” (Filiberto Gómez, citado en Montemayor 1999, p. 144).

${ }^{9}$ Resistencia innegable cuyo origen han sido probablemente las estrategias de desarrollo y el despojo de su territorio, todo emprendido principalmente por agentes externos. Para un recuento de las primeras -minería, turismo y narcotráfico- en toda la región Tarahumara puede verse en Sariego (2002, pp. 2011-219). Y, sobre el despojo de tierras, una muestra puede verse en Meza (2001). Al tratar la histórica tensión vivida, y permanente aún, entre mestizos y rarámuri de Bakiachi, en este trabajo de corte etnohistórico, esta autora prueba cómo "las escasas tierras cultivables [en la sierra Tarahumara] han sido acaparadas por los mestizos, asentados en la sierra desde la época colonial" (p. 37). socialización. Revista de Investigaciones Folclóricas, 16, 77-86. Recuperado de http://mail.arte.com.ar/ifa/publicaciones/RIF16. pdf\#page $=77$.

Bennett, C. \& Zingg, R. (1978 [1935]). Los tarahumaras: Una tribu india del norte de México. México: Instituto Nacional Indigenista.

Bergson, H. (2011 [1900]). La risa: Ensayo sobre el significado de la comicidad. Madrid: Sarpe. Recuperado de https://www.academia. edu/21062656/La_risa_Henri_Bergson?auto=download.

Bonfiglioli, C. (1995). Fariseos y matachines en la Sierra 
Tarahumara, entre la pasión de Cristo, la transgresión cómico sexual y las danzas de conquista. México: Instituto Nacional Indigenista.

Bourdieu, P., Chamboredon, J. \& Passeron, J. (2008). El oficio del sociólogo, presupuestos epistemológicos. México: Siglo XXI.

Brambila, D. (1976). Diccionario rarámuri-castellano. México: Obra Nacional de la Buena Prensa.

Cipolletti, M. (2000-2001). Expresión, humor y crítica oculta en la historia de vida de un chamán amazónico. Société suisse des Américanistes / Schweizerische Amerikanisten-Gesellschaft Bulletin, 64-65, 205-213.

Galinier, J. (2001). Una mirada detrás del telón: Rituales y cosmovisión entre los otomíes orientales. En Broda, J. y Félix Báez, J. (coords.). Cosmovision, ritual e identidad de los pueblos de Mexico (pp. 453-484). México: Biblioteca Mexicana, Consejo Nacional para la Cultura y las Artes, Fondo de Cultura Económica.

Geertz, C. (2005 [1973]). La interpretación de las culturas. Barcelona: Gedisa.

González, L. (1982). Tarahumara: La sierra y el hombre. Chihuahua: Camino.

(1987). Crónicas de la Sierra Tarahumara. México:

Secretaría de Educación Pública.

Huizinga, J. (2007). Homo ludens. Madrid: Alianza, Emecé.

INEGI (2009). Carta topográfica de "Narárachi (Alto Río Conchos) G13A23", esc. 1: 50. 000. México: Instituto Nacional de Estadística y Geografía.

(2015). Encuesta Intercensal. Aguascalientes: Instituto Nacional de Estadística y Geografía. Recuperado de http://www3.inegi. org.mx/sistemas/TabuladosBasicos/Default.aspx?c=27303\&s=est.

Infante, C. (2008). Poder, tensión y caricatura: Una aproximación a la teoría del humor. Dialogía, Revista de Lingüística, Literatura y Cultura, 3, 245-272.

Jáuregui, E. (2008). Universalidad y variabilidad cultural de la risa y el humor. AIBR, Revista de Antropología Iberoamericana, 3(1), 46-63.

Kennedy, J. (1963). Texgüino complex: The role of beer in Tarahumara culture. American Anthropologist, 65, 620-640. (1970a). Bonds and laughter among the Tarahumara Indian. Latin American Studies, 14, 36-68.

(1970b). Inápuchi: Una comunidad tarahumara gentil.

México: Instituto Indigenista Interamericano.

Kravitz, S. (1977). London jokes and ethnic stereotypes. Western Folklore, Western States Folklore Society, 36, 275-301. Recuperado de https://www.jstor.org/stable/1499195.

Lagrou, E. (2006). Rir do poder e o poder do riso nas narrativas e performances kaxinawa. Revista de Antropología, 49(1), 55-90.

Lionnet, A. (1972). Los elementos de la lengua tarahumara. México: Universidad Nacional Autónoma de México.

López, S. (2008). Humor y poder. AlBR, Revista de Antropología Iberoamericana, 3(1), 64-94.

Lumholtz, C. (1981 [1902]). El México desconocido, 2 tomos. México: Instituto Nacional Indigenista.

Llera, J. (2004). La investigación en torno al humor verbal. Revista de Literatura, LXVI(132), 527-535. Recuperado de: http:// revistadeliteratura.revistas.csic.es/index.php/revistadeliteratura/ article/viewFile/132/143.

Meza, M. (2001). San José Baqueachi: Historia de un ejido tarahumara que se resiste al despojo de sus tierras. México: Instituto Chihuahuense de la Cultura.

Merrill, W. (1992). Almas rarámuri. México: Consejo Nacional para la Cultura y las Artes, Instituto Nacional Indigenista.

Montemayor, C. (1999). Los Tarahumaras. Pueblo de estrellas y barrancas. México: editorial Aldus.

Oring, E. (2008). Humor in anthropology and folklore. En Raskin, V. \& Ruch, W. (Eds.), The primer of humor research (pp. 183-210). Berlín y Nueva York: Mouton de Gruyter.

Rodríguez, A. (2009). Reunión y bebida colectiva entre los rarámuri, (la tesgüinada) una institución difícil de comprender. En Orozco, V. (Coord.), Chihuahua Hoy 2009 (pp. 91-135). México: Universidad Autónoma de Ciudad Juárez, Universidad Autónoma de Chihuahua, Instituto Chihuahuense de la Cultura.

(2010). ¿Por qué piden el bautismo católico los rarámuri del Alto Río Conchos? Estudios Mesoaméricanos Revista del Posgrado en Estudios Mesoamericanos-Universidad Nacional Autónoma de México, (9), 63-74.

(2013). Praxis religiosa, simbolismo e historia de los rarámuri del Alto Río Conchos. Quito: Abya-Yala.

Salinas, M. (1991). La risa y el buen humor de los chilenos. Universidad ARCIS. Documento de Trabajo (pp. 9-17.) Recuperado de http://admisiononline.uft.cl/bitstream/handle/20.500.12254/1324/la\%20 risa\%20y\%20el\%20buen\%20humor\%20de\%20los\%20chilenos\%20 2003. pdf? sequence $=1$ \&isAllowed $=y$

(1996). Risa y cultura en Chile. Biblioteca Virtual de CLACSO. Universidad ARCIS. Documento de Trabajo № 1 , agosto 1996. Recuperado de http://biblioteca.clacso.edu.ar/Chile/ di-uarcis/20120927095411/salinas.pdf.

Sapir, E. (1956). Culture, language and personality: Selected essays. Berkeley y Los Ángeles: G. B. Mandelbaum, University of California Press.

Sariego, J. (2002). El indigenismo en la Tarahumara: Identidad, comunidad, relaciones interétnicas y desarrollo en la Sierra de Chihuahua. México: Instituto Nacional Indigenista, Consejo Nacional para la Cultura y las Artes, Instituto Nacional de Antropología e Historia.

Tobón, M. (2016). Reírse ante la guerra: Las bromas como actuación política entre los Muina, Amazonia colombiana. Revista Mexicana de Sociología, 78, 179-202.

Torres Sánchez, M. (1997-1998). Teorías lingüísticas del humor verbal. Pragmalingüística 5-6, 435-448.

Valiñas, L. (2002). Reflexiones en torno a las lenguas guazapar y tarahumara coloniales. Anales de Antropología, 36, 249-282.

Velasco, P. (2006 [1983]). Danzar o morir, religión y resistencia a la dominación en la cultura tarahumara. Guadalajara: Instituto Tecnológico y de Estudios Superiores de Occidente, Universidad Iberoamericana, Complejo Asistencial Santa Teresita.

Wagner, R. (2018 [1975]). La invención de la cultura. (traducción de Pedro Pitarch). México: Nola editores. 